\title{
Review of: "Past and future trends of civil airport emissions in China, from 2010 to 2030"
}

\author{
Dongsheng Chen ${ }^{1}$ \\ 1 Beijing University of Technology
}

Potential competing interests: The author(s) declared that no potential competing interests exist.

\section{Summary}

This paper proposes a method to investigate the trends of China's civil airport emissions from 2010 to 2030. In addition, it quantifies the impact of COVID-19 on emissions from civil airports. This paper can be used as a reference for the study of civil airport emissions and the impact of the epidemic on pollutant emissions. Generally speaking, this paper is suitable for publication in this journal.

\section{Major comments}

1. Why did you choose only seven airports to represent all the airports in China? Can you choose more airports? Please elaborate on the data sources of these seven airports.

2. Ground support equipment, boilers, oil depots, and parks are also sources of airport pollutants. Why not consider their emissions in this study?

3. The 3.3 Comparison of estimated emissions section of the paper only qualitatively analyzes the difference from the previous study, which is not enough. Please combine the research methods of each paper to make a quantitative analysis of this difference and explain the reasons.

4. There are many problems in the reference section, please revise this section carefully.

\section{Minor comments}

Line 26-27: Scientific notation for numbers would be much easier to read.

Line 36-37: Does the initial need to be capitalized? What is the correct expression order of "Central South"? Line 48: Please give details when all abbreviations appear for the first time.

Line 58: This sentence should be "Establishing a pollutant emission inventory is the basis of establishing effective measures for developing pollution control strategies"?

Line 86-91: I needed to read this section several times to decipher what was being done. Please rewrite this section.

Line 103-105: Please add the corresponding references.

Line115-121: Check the full names of the seven airports.

Line144: Incomplete references.

Line191: The format of units is not uniform.

Line198: The format of the equation is not uniform. 
Line 320-321: There is a word order problem with this sentence.

Line 618-622: The picture is not clear enough 